\title{
TIPIFICACIÓN DE COMERCIALIZADORES DE BERENJENA EN ZONAS PRODUCTORAS DEL CARIBE COLOMBIANO
}

\section{TYPING OF EGGPLANT TRADERS IN PRODUCTION AREAS OF THE COLOMBIAN CARIBBEAN}

\author{
Ender Correa ${ }^{1}$, Hermes Araméndiz², Leandro Azeredo ${ }^{3}$, Claudia Pombo ${ }^{3}$, Carlos Cardona² \\ Recibido para evaluación: Junio 8 de 2010 - Aceptado para publicación: Noviembre 30 de 2010
}

\begin{abstract}
RESUMEN
La berenjena es una de las principales hortalizas de los departamentos de Córdoba y Sucre. El objetivo fue conocer las características de la comercialización en el Caribe colombiano e identificar grupos con posibilidades de éxito de cara a los acuerdos de libre comercio. El estudio se realizó mediante encuestas con opción de respuesta múltiple dirigida a 20 comercializadores mayoristas de berenjena, indagando características del comercializador, del producto, de su manejo y de la comercialización. Utilizando técnicas de análisis multivariado se identificaron cinco grupos de comercializadores que registran infraestructura y organización deficiente para el comercio del producto. El grupo I representa el $20 \%$ de los comercializadores, poseen escolaridad básica primaria y pierden entre el $5 \%$ y $10 \%$ del producto por mala conservación; el grupo II agrupa al 65\%, combinan educación básica primaria y secundaria, pierden entre el $15 \%$ y $20 \%$ y conservan el producto en bolsas plásticas; el grupo III representa el 5\%, presenta escolaridad básica primaria, desconoce la vida útil del fruto y sus pérdidas ascienden al 20\%; el grupo IV está conformado por un 5\%, tiene mayor escolaridad, conserva en refrigeración, sus pérdidas oscilan entre $10 \%$ y $15 \%$ y presenta deficiente control de calidad al adquirir el producto, finalmente, el grupo $\mathrm{V}(5 \%)$ presenta escolaridad básica secundaria y comercializa en un mismo día con pérdidas del 5\%. Este trabajo permite concluir que el sistema de comercialización de la berenjena en la región caribe es heterogéneo y artesanal, que lo torna no competitivo en los mercados nacionales e internacionales especializados.
\end{abstract}

Palabras clave: Solanum melongena, mercados, calidad de fruto, análisis multivariado

\begin{abstract}
Eggplant is one of the main vegetables in the departments of Córdoba and Sucre. The objective was to determine the marketing in the Colombian Caribbean and identify groups that likely would be successful in front of free

\footnotetext{
1'Ingeniero Agrónomo, Estudiante de Maestría, Universidad de Córdoba endermanz@hotmail.com ${ }^{2}$ Ingeniero Agrónomo, Ph.D. Profesor titular, Universidad de Córdoba, Montería - Colombia haramendiz@hotmail.com ${ }^{2}$ Ingeniero Agrónomo, M.Sc. Profesor titular, Universidad de Córdoba, Montería - Colombia ceca24@ hotmail.com ${ }^{3}$ Ingenieros Agronómos, Ph.D. UENF, Av. Alberto Lamego, 2000, Parque Califórnia, 28013-602 Campos dos Goytacazes RJ. Brasil
} 
trade agreements. The study was conducted through surveys with multiple choice options addressed to 20 eggplant wholesale traders. Characteristics of the marketer, the product, its management and marketing were investigated. Using techniques of multivariate analysis five groups of traders were identified, reported poor infrastructure and organization to trade the product. Group I represents $20 \%$ of marketers that possess basic primary education and lose between $5 \%$ and $10 \%$ of the product by poor maintenance; group II comprises $65 \%$, combined primary and secondary basic education, they lose between $15 \%$ and $20 \%$ and keep the product in plastic bags; group III represents the $5 \%$ with primary basic education, unknown the life cycle of the product and loses $20 \%$; group IV is composed by $5 \%$, they have better schooling, kept the product under refrigeration and loses between $10 \%$ and $15 \%$. This group has poor quality control when purchase the product; finally the group $\mathrm{V}$ represents $5 \%$, has basic secondary education and markets in one day with losses of 5\%. This work allows us to conclude that the eggplant marketing system in the Caribbean region is heterogeneous and craftsmanship, it becomes uncompetitive in domestic and international specialized markets

Key Words: Solanum melongena, markets, fruit quality, multivariate analysis

\section{INTRODUCCIÓN}

La berenjena es una especie hortícola de importancia económica y social en el Caribe colombiano, por el impacto en la generación de empleo familiar (IWS 2011), especialmente en los departamentos de Córdoba y Sucre, cuya área cultivada en el 2009 fue de 141 ha, labor realizada por pequeños productores, en áreas que no superan los $10.000 \mathrm{~m}^{2}$ y en su conjunto comercializaron una producción de 1310 t que representa el 38, $35 \%$ de la producción nacional (Agronet 2009).

El gobierno colombiano es consciente de la necesidad de reducir la brecha tecnológica en este cultivo y estar a la par de México, Honduras y República Dominicana, que ocupan un lugar de privilegio en las exportaciones a nivel mundial (FAO 2010), cuyo mercado entre 2002 - 2006 registró un crecimiento del 12\% (Mineco - Unión Europea 2010) a causa del crecimiento en el mercado de las hortalizas, cuya tasa promedio fue del 4,1\%, por el mejoramiento de los ingresos, cambios del estilo de vida traído por la urbanización y mudanzas en las estructuras familiares, que han producido cambios en la dieta a lo largo del mundo (Ferrato y Mondino 2008).

La comercialización agrícola es un proceso que permite que los productos Ileguen a los consumidores localizados en zonas urbanas distantes de las áreas de producción, de tal manera que el número de operaciones y funciones que se llevan a cabo, determinan el grado de complejidad del proceso. De esta forma, se puede decir que la comercialización es el conjunto de procesos o etapas que deben superar los productos en el flujo de traslado desde el productor hasta el consumidor final (Mendoza 2002).

En Colombia, a pesar de contar con recursos naturales muy valiosos, favorables para el cultivo de hortalizas; no se cuenta con un sistema de mercado organizado que permita comprender la complejidad de los productos, ni que oriente a la creación de una industria de vanguardia ajustada a la realidad colombiana y lista para enfrentar los cambios con respecto a la comercialización de productos (Salinas y Raigosa 2005). 
En los territorios donde existe una agricultura empresarial con esta especie, como la "Berenjena de Almagro" en España, esta actividad ha generado dinamismo económico local, posicionamiento en el mercado y desarrollo para las cinco empresas agrícolas existente en su área de influencia, principalmente a través de su vinculación a la agroindustria, que son actividades motores del crecimiento sectorial (Almansa 2011). Sin embargo, donde más predominan este tipo de unidades productivas es donde más se carece de capacidad empresarial, especialmente en los cultivos asociados a la economía campesina, cuya dinámica es empobrecedora y posee brechas crecientes respecto de la agricultura con mayor potencial como lo anotan Jara et al. (2009).

Minagri (2000) destaca que las empresas agrícolas son uno de los principales agentes del mundo privados llamados a impulsar la transformación productiva necesaria para posicionar a una región como una potencia agroalimentaria. Sin embargo, y a diferencia de lo que ocurre en otros sectores, en la agricultura es particularmente difícil concebir a estos agentes como una entidad homogénea, con las mismas capacidades para adaptarse a las crecientes exigencias que imponen los nuevos mercados agroalimentarios globales y el mismo potencial para beneficiarse de las políticas e instrumentos de fomento a la innovación, propuestos por los gobiernos (Jara et al. 2009).

La agricultura campesina se ha caracterizado históricamente por poseer mínimo acceso a crédito, baja dotación de capital, limitado uso de tecnologías, escasa conectividad digital y bajos niveles de capital humano. Además de estos factores propios de la unidad productiva, la pequeña agricultura muchas veces se encuentra localizada en sectores con una deficiente dotación de recursos naturales, mientras que los sectores más dinámicos e integrados con las cadenas productivas y comerciales denotan la tendencia contraria (Cordero-Salas et al. 2003).

El agrupamiento de los comercializadores de un producto agrícola como actores en un sistema de producción, es posible a través de las técnicas de análisis multivariado considerando las diferencias relacionadas con el tipo de comercializador, manejo y calidad del producto, etc; de tal manera que ello permita establecer por algún criterio grupos, los cuales deben ser homogéneos en su interior y contrastantes entre si, con el propósito de identificar con más seguridad los más divergentes en la cadena del mercado y problemática relevante en ellos. Por tal razón, las técnicas de análisis estadístico multivariado constituyen una herramienta idónea para la tipificación y clasificación de sistemas de producción agropecuarios; estos sistemas presentan varios componentes o subsistemas interactuando en el tiempo y en el espacio, así como en relación con suprasistemas de diversa naturaleza. Además, los métodos estadísticos multivariados permiten operacionalizar el sentido heurístico de la teoría de sistemas, aplicada al problema de clasificar y tipificar sistemas de fincas (Escobar y Berdegué 1990).

La caracterización y tipificación de grupos similares en el campo agropecuario a través del análisis multivariado ha sido aplicada en la caracterización de comercializadores de vinos (Coca y Solís 2008), cítricos (Poole y 
Gomis 2000; Rios et al. 2007) y, sistemas de producción de pasifloras (Miranda y Carranza 2010), hortalizas (Hang et al. 2010), frijol (Miranda 1990) y Maíz (Espinosa et al. 1990) entre otros.

El objetivo del presente estudio fue conocer las características de la comercialización de la berenjena en el Caribe colombiano e identificar grupos con posibilidades de transitar con éxito hacia la modernización o, sumirse en el rezago del crecimiento y desarrollo sectorial ante las perspectivas de tratados de libre comercio.

\section{MATERIALES Y MÉTODOS}

El estudio se realizó en el año 2008 en las principales áreas productoras de berenjena de los departamentos de Córdoba y Sucre. Se estudiaron aspectos inherentes a características del comercializador, características del producto, manejo del producto y comercialización del producto, mediante entrevistas realizadas a diferentes actores relacionados con la comercialización mayorista en los municipios de mayor importancia en el mercado de la berenjena. Las encuestas, consistieron en preguntas elaboradas con la participación de funcionarios de entidades ligadas al sector agrícola, como Universidad de Córdoba, Corpoica, Secretaria de Educación del Departamento de Sucre, Secretaría de Agricultura del Departamento de Córdoba, Instituto Colombiano Agropecuario, Hortisinú y productores que realizan su propia comercialización, mediante entrevistas realizadas a distintos actores vinculados con el comercio mayorista. Las encuestas, en su mayoría consistieron en cuestionarios que aceptaban respuestas con opción múltiple.

El universo de estudio fue conformado por 20 comercializadores, a quienes se les realizó la entrevista en los mercados, lugar de producción y visitas domiciliarias, a través de 27 preguntas consignadas en la tabla 1.

Tabla 1. Aspectos sobre la comercialización de berenjena en Córdoba y Sucre

\begin{tabular}{|c|c|c|}
\hline ASPECTOS & VARIAI & \\
\hline $\begin{array}{l}\text { Características del } \\
\text { comercializador }\end{array}$ & $\begin{array}{l}\text { Departamento } \\
\text { Municipio } \\
\text { Escolaridad }\end{array}$ & Experiencia en la comercialización \\
\hline $\begin{array}{l}\text { Características del } \\
\text { producto }\end{array}$ & $\begin{array}{l}\text { Presentación del producto } \\
\text { Color del fruto } \\
\text { Forma del fruto }\end{array}$ & $\begin{array}{l}\text { Tamaño del fruto } \\
\text { Variedades de mayor demanda } \\
\text { Perdidas poscosecha }\end{array}$ \\
\hline $\begin{array}{l}\text { Manejo del } \\
\text { Producto }\end{array}$ & $\begin{array}{l}\text { Vida útil poscosecha } \\
\text { Método de conservación del producto } \\
\text { Tipo de empaque }\end{array}$ & $\begin{array}{l}\text { Factores que causan perdidas } \\
\text { poscosecha } \\
\text { Destino de la berenjena de desecho }\end{array}$ \\
\hline $\begin{array}{l}\text { Características de la } \\
\text { comercialización }\end{array}$ & $\begin{array}{l}\text { Obtención del producto } \\
\text { Peso por bulto } \\
\text { Frutos por bulto } \\
\text { Bultos comercializados por mes } \\
\text { Días por semana de comercialización } \\
\text { Tiempo de venta }\end{array}$ & $\begin{array}{l}\text { Principales clientes } \\
\text { Factores que limitan la comercialización } \\
\text { Mes de mayores ganancias } \\
\text { Proveedor del empaque } \\
\text { Unidad de comercialización } \\
\text { Mercado de destino }\end{array}$ \\
\hline
\end{tabular}


Para el análisis de la información obtenida y lograr la tipificación de los comercializadores, se realizó el análisis estadístico que consistió en la obtención de una matriz de desemejanza con las variables cualitativas y cuantitativas categorizadas y así obtener el índice de complemento de Jaccard (Cruz y Carneiro 2003), con la previa transformación de los datos a binario. Este índice se basa en la siguiente formula:

$D_{i j}=\frac{b+c}{a+b+c}$

Donde:

Dij= distancia entre los comercializadores i y $\mathrm{j}$, sin considerar el número de características $\mathrm{a}=$ valor que cuantifica el número de coincidencia de tipo $1-1$ para cada par de comercializadores

$b=$ valor que cuantifica el número de no coincidencia de tipo 1 - 0 para cada par de comercializadores

$\mathrm{c}=$ valor que cuantifica el número de no coincidencia de tipo $0-1$ para cada par de comercializadores

Con los valores obtenidos de disimilitud y con el apoyo del programa Genes (Cruz 2004), se realizó la agrupación de los comercializadores por el método de Tocher (Rao 1952), el cual tiene como objetivo la formación de grupos, considerando que dentro de cada grupo debe de existir la mayor homogeneidad posible $y$ entre grupos alta heterogeneidad.

De igual manera, se aplicó el método de agrupación de pares no ponderados con medias aritméticas (UPGMA); que es un método no ponderado de formación de grupos, basado en el uso de las medias aritméticas de desemejanza, que evitan caracterizar la disimilitud por valores extremos (máximo o mínimo) entre los individuos considerados en el estudio. De esta manera, el dendograma es construido con los comercializadores de mayor semejanza y la distancia entre dos grupos es dada por la media del conjunto, cuyos elementos representan las distancias entre los pares de comercializadores (i y k), (i y l), (j y k) y (j y l) (Cruz y Carneiro 2003). La confiabilidad del agrupamiento UPGMA, fue realizada a través del coeficiente de correlación cofenética (CCC), que de acuerdo a Mohammdi y Prasanna (2003), cuando se obtienen altos valores, ello refleja mejor representación y mayor eficiencia del método de agrupamiento. En este sentido (Rohlf 1992), señala que valores $0,9 \leq r$, señalan un excelente agrupamiento; $0,8 \leq r<0,9$ buen agrupamiento; $0,7 \leq r<0,8$ regular agrupamiento; $r<0,7$, mal agrupamiento.

Por otra parte, se realizó el análisis de coordenadas principales (CP) desarrollado por Gower (1966) con el propósito de reducir la dimensión de los datos e interpretar las similitudes y disimilitudes entre los individuos de manera simple. Para ello, las disimilaridades entre individuos se mide mediante una distancia cualquiera que no tiene que tener una interpretación física concreta, el resultado final son las coordenadas de los individuos en un espacio euclídeo en el que la distancia es la euclídea usual con la interpretación física usual (Cuadras 1981; Vicente 2011).

\section{RESULTADOS Y DISCUSIÓN}

El análisis de disimilaridad realizado por el método de Tocher (Tabla 2), permitió la 
Tabla 2. Matriz de disimilaridad basada en el índice de Jaccard

\begin{tabular}{|c|c|c|c|c|c|c|c|c|c|c|c|c|c|c|c|c|c|c|c|c|}
\hline Comercializadores & 1 & 2 & 3 & 4 & 5 & 6 & 7 & 8 & 9 & 10 & 11 & 12 & 13 & 14 & 15 & 16 & 17 & 18 & 19 & 20 \\
\hline 1 & 0.00 & & & & & & & & & & & & & & & & & & & \\
\hline 2 & 0.58 & 0.00 & & & & & & & & & & & & & & & & & & \\
\hline 3 & 0.58 & 0.39 & 0.00 & & & & & & & & & & & & & & & & & \\
\hline 4 & 0.45 & 0.54 & 0.59 & 0.00 & & & & & & & & & & & & & & & & \\
\hline 5 & 0.60 & 0.54 & 0.48 & 0.56 & 0.00 & & & & & & & & & & & & & & & \\
\hline 6 & 0.74 & 0.70 & 0.57 & 0.81 & 0.69 & 0.00 & & & & & & & & & & & & & & \\
\hline 7 & 0.65 & 0.55 & 0.43 & 0.61 & 0.59 & 0.57 & 0.00 & & & & & & & & & & & & & \\
\hline 8 & 0.56 & 0.59 & 0.59 & 0.55 & 0.52 & 0.69 & 0.50 & 0.00 & & & & & & & & & & & & \\
\hline 9 & 0.79 & 0.76 & 0.75 & 0.82 & 0.83 & 0.77 & 0.82 & 0.85 & 0.00 & & & & & & & & & & & \\
\hline 10 & 0.63 & 0.78 & 0.71 & 0.62 & 0.71 & 0.79 & 0.69 & 0.70 & 0.74 & 0.00 & & & & & & & & & & \\
\hline 11 & 0.63 & 0.50 & 0.53 & 0.55 & 0.60 & 0.76 & 0.58 & 0.48 & 0.81 & 0.65 & 0.00 & & & & & & & & & \\
\hline 12 & 0.63 & 0.73 & 0.65 & 0.62 & 0.70 & 0.76 & 0.69 & 0.69 & 0.81 & 0.58 & 0.56 & 0.00 & & & & & & & & \\
\hline 13 & 0.80 & 0.84 & 0.82 & 0.83 & 0.78 & 0.84 & 0.83 & 0.82 & 0.76 & 0.70 & 0.82 & 0.78 & 0.00 & & & & & & & \\
\hline 14 & 0.81 & 0.80 & 0.75 & 0.81 & 0.78 & 0.86 & 0.80 & 0.82 & 0.68 & 0.79 & 0.76 & 0.83 & 0.82 & 0.00 & & & & & & \\
\hline 15 & 0.79 & 0.78 & 0.68 & 0.81 & 0.74 & 0.76 & 0.74 & 0.71 & 0.70 & 0.81 & 0.78 & 0.83 & 0.84 & 0.32 & 0.00 & & & & & \\
\hline 16 & 0.73 & 0.78 & 0.73 & 0.74 & 0.79 & 0.84 & 0.82 & 0.76 & 0.73 & 0.79 & 0.78 & 0.81 & 0.83 & 0.30 & 0.33 & 0.00 & & & & \\
\hline 17 & 0.80 & 0.87 & 0.78 & 0.85 & 0.78 & 0.74 & 0.77 & 0.78 & 0.72 & 0.82 & 0.87 & 0.84 & 0.80 & 0.51 & 0.38 & 0.49 & 0.00 & & & \\
\hline 18 & 0.63 & 0.75 & 0.68 & 0.62 & 0.62 & 0.86 & 0.65 & 0.59 & 0.81 & 0.60 & 0.61 & 0.65 & 0.78 & 0.75 & 0.72 & 0.71 & 0.82 & 0.00 & & \\
\hline 19 & 0.73 & 0.74 & 0.69 & 0.68 & 0.71 & 0.75 & 0.66 & 0.68 & 0.86 & 0.54 & 0.57 & 0.51 & 0.72 & 0.90 & 0.85 & 0.83 & 0.90 & 0.59 & 0.00 & \\
\hline 20 & 0.63 & 0.72 & 0.60 & 0.66 & 0.54 & 0.71 & 0.62 & 0.68 & 0.82 & 0.50 & 0.60 & 0.57 & 0.75 & 0.76 & 0.76 & 0.78 & 0.77 & 0.67 & 0.70 & 0.00 \\
\hline
\end{tabular}

formación de cinco grupos (Tabla 3). Esto corroboró la existencia de divergencia en los comercializadores existentes en los departamentos de Córdoba y Sucre, a pesar de ser una zona geográfica bastante homogénea en lo pertinente a sus sistemas de producción. Así mismo, resalta la necesidad de generar asociatividad entre tipos de comercializadores, con el fin de lograr mejores condiciones de comercialización, productivas y tecnológicas de almacenamiento como lo señalan Bustamante y Gálvez (2010).

El grupo I representa el $20 \%$ de los comercializadores y presenta las siguientes características: su área geográfica de influencia es el departamento de Sucre en los municipios de Corozal, Sampués y Sincelejo; con un nivel de escolaridad de básica primaria; la mala calidad que presentan los frutos de berenjena que llegan para su comercio obedece principalmente a daños causados por insectos, enfermedades, actividades mecánicas e igualmente frutos con deshidratación a causa de la mala conservación, que conduce a pérdidas que oscilan entre el $5 \%$ y $10 \%$; la mayor rentabilidad de su actividad se concentra en el mes de marzo; usan empaque ralos; comercializan las variedades criollas: lila, negra, pompa y cacho de chivo de frutos de tamaño grande (20 a $35 \mathrm{~cm}$ de longitud) y forma cilíndrica; cuyo principales destinos corresponden a los mercados de Sincelejo, Barranquilla y Cartagena y experiencia en el mercado preponderante oscila entre 20 y 30 años.

Tabla 3. Grupos de comercializadores de berenjena establecidos por el método de Tocher, con base en la disimilaridad existente entre ellos.

\begin{tabular}{llllllllllllllll}
\hline I & 14 & 15 & 16 & 17 & & & &
\end{tabular}


El grupo II agrupa el 65\% de los encuestados, los cuales pertenecen al departamento de Córdoba, destacando los municipios de Planeta Rica, Cereté y Montería; con nivel de escolaridad básica primaria - secundaria; experiencia de mercado de 10 años; las enfermedades y plagas constituyen el factor limitante de la calidad del producto para su venta en el mercado; conservan los excedentes en bolsas plásticas; la unidad de mercado son bultos de aproximadamente $50 \mathrm{~kg}$ que se empacan en sacos de fique con pérdidas entre el $15 \%$ y $20 \%$; para éstos diciembre representa el mes de mayor beneficio económico y sus principales destinos son Sincelejo, Montería, Cartagena, Barranquilla y ciudades intermedias. Así mismo, las variedades de mayor comercialización son las de color lila, morada, carne y negra. Al respecto, el SIPSA (2008) reporta que las variedades de mayor comercialización en el mercado de Montería son las que presentan una pigmentación morada intensa, casi negra (morada) y moradas más clara (lila), considerándose esta última como la de mejor calidad, por presentar sabor más suave y mayor cantidad de pulpa.

El grupo III está compuesto por un solo comercializador que representa el $5 \%$ de la muestra con características divergentes a los otros grupos, se destaca por ser del departamento de Córdoba, oriundo de Cereté; con nivel de escolaridad de básica primaria y experiencia de mercado entre 21 y 30 años, vendiendo alrededor de 200 bultos por mes, equivalentes a 10 toneladas; desconoce la vida útil del producto, razón por la cual las perdidas alcanzan el nivel del 20\%; su principal cliente es el consumidor final; las lluvias su mayor dificultad para vender, diciembre es el mes de mayor rentabilidad; el fruto apetecido por el mercado es alargado y de tamaño mediano de las variedades de colores lila y morado en el mercado de Montería.

El grupo IV al igual que el anterior está conformado por un comercializador que representa el 5\% de la muestra en estudio, oriundo del municipio de Montería en el departamento de Córdoba; posee escolaridad profesional; experiencia de 11 - 20 años; conserva en refrigeración; comercializa a cadenas de supermercado; unidad de comercialización es el kilo, lo que le permite vender entre 10 y 20 toneladas por mes en sacos tipo tres rayas, que es de mejor consistencia y presentación al mercado; marzo es el mes de mayor rentabilidad, siendo el tipo de fruto preferidos aperado y grande; las pérdidas oscilan entre un $10 \%$ y $15 \%$ a causa de daños mecánicos y tamaño pequeño del fruto; siendo las variedades de color lila, morado y negro las preferidas por sus clientes.

El grupo $\mathrm{V}$ posee un solo comercializador que representa el $5 \%$ de la población; es nativo de Montería en el departamento de Córdoba; el nivel de escolaridad corresponde a básica secundaria; experiencia de 10 años en el mercado, lo hace por kilos y en un mismo día todo lo que recibe con el fin de minimizar las pérdidas que son del nivel del 5\%, ya que no usa métodos de conservación; el principal limitante está representado por enfermedades en poscosecha; junio el mes de mayor rentabilidad; el fruto preferido es alargado y pequeño de los cultivares lila y negro.

De acuerdo al método UPGMA (Figura 1), se puede inferir que existe divergencia entre los 20 


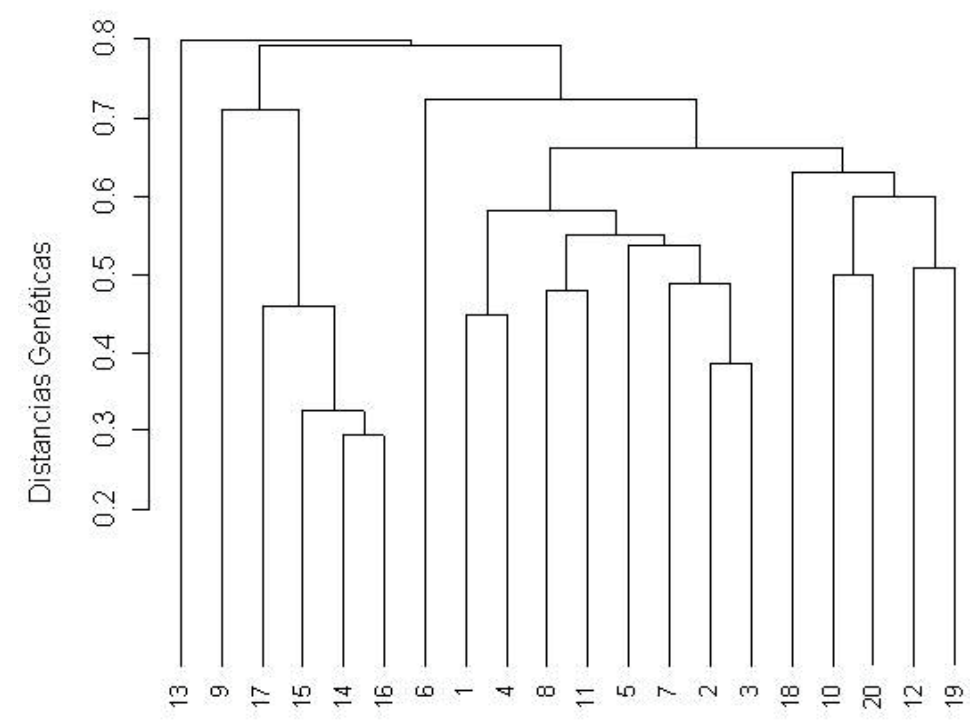

Figura 1. Dendograma de disimilaridad de grupos de comercializadores de berenjena, obtenido por el método UPGMA.

comercializadores de berenjena encuestados en los departamentos de Córdoba y Sucre, debido a la delimitación de cinco grupos considerando el corte en el eje $\mathrm{x}$ al $70 \%$ de la distancia relativa entre los comercializadores. El coeficiente de correlación cofenética (CCC) de dicho dendrograma resultó de elevada magnitud $(r=0,81), \quad p<0,01$, evidenciando consistencia y excelente agrupamiento como lo indican (Rohlf 1992; Mohammdi y Prasanna 2003).

El método de coordenadas principales (Figura 2) que es la representación geométrica de los comerciantes de acuerdo a su distancia de similaridad, condujo a resultados similares en la ubicación de los grupos en un plano geográfico; pudiéndose apreciar los cinco grupos en forma clara, con características de experiencia en el mercado, escolaridad, porcentaje de pérdidas diferentes en razón al tipo de almacenamiento usado para preservar su calidad.

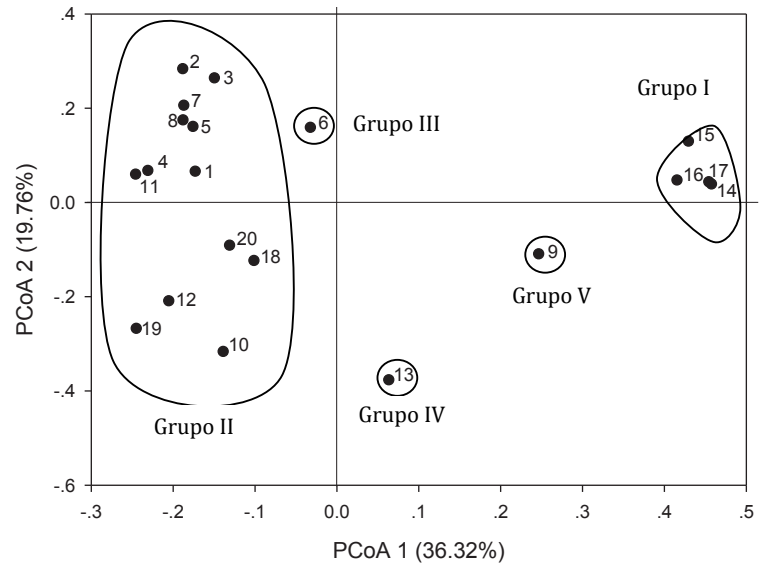

Figura 2. Distribución de los comercializadores de berenjena en el análisis de coordenadas principales.

Lo antes anotado, destaca que el método de agrupamiento de Tocher, el método de coordenadas principales y el método de agrupación de pares no ponderados con medias aritméticas UPGMA, fueron concordantes y eficaces en la conformación de grupos de acuerdos a sus características económicas, sociales, naturales y técnicas. 
Comparando los cinco grupos definidos por las circunstancias sociales, económicas, tecnológicas y de infraestructura, a excepción del grupo IV, que posee un nivel de escolaridad alto y aplica técnicas de refrigeración, los demás grupos presentan debilidades en cuanto a volumen de comercialización, deficiente presentación del producto por el tipo de empaque y método de conservación usado que los torna frágil para proyectarlos como empresarios que le otorgue dinámica a la economía regional como la berenjena de Almagro (Almansa 2011) e incursionar en un mundo donde los tratados de libre comercio, globalización y competitividad, representan un reto como lo hacen México, República Dominicana y Honduras con esta hortaliza (IICA 2007; Guajardo y Ríos 2009; FAO 2010); dado que usan empaques inadecuados como sacos de fique o paperos que influyen en la calidad del producto que llega al comercio local, además de la no aplicación de buenas prácticas de poscosecha como el lavado, sanitización y revestimiento de frutos, selección por tamaño, forma, color y ausencia de daño mecánico que redundan en la buena presentación del producto y el cumplimiento de los requerimientos fitosanitarios de los mercados especializados (Guajardo y Ríos 2009). Lo recomendado es el uso de cajas de cartón parafinado, envolviendo los frutos individualmente en bolsas $u$ hojas de papel para proteger el aspecto brillante y liso (IICA 2007) y bajo refrigeración a $12{ }^{\circ} \mathrm{C}$ y una vida útil de 10 días (Dos Santos y Henz 1993).

Lo antes anotado es reflejo de un sistema de producción que aún carece de adopción de tecnologías y políticas de asociatividad de productores $y / 0$ cooperativismo que permitan la estructuración de unidades de producción mucho más fuertes y así, reducir las diferencias tecnológicas como lo anotan Jara et al. (2009) y de esta manera ofertar un producto acorde a las normas establecidas por bloques comerciales de países como volumen, calidad y continuidad de la exportación a través del tiempo. Además, Guajardo y Ríos (2009), anotan que los cambios en la política de mercado agrícolas que afectan variables económicas como precios, producción, consumo, importaciones, exportaciones e ingresos de los productores. De igual manera, dada sus limitaciones empresariales por el capital humano que lo integra es un obstáculo para proyectar al país en el mercado internacional y ello amerita, no solo la generación de conocimientos que mejoren los niveles de producción, sino la aplicación de buenas prácticas agronómicas asociadas con buenas prácticas de proceso, que garanticen éxito de la oferta a los mercados nacionales e internacionales, como lo viene logrando México, que es el segundo exportador de berenjena a nivel mundial.

\section{CONCLUSIONES}

El sistema de comercialización de berenjena en la región caribe es heterogéneo y artesanal, que lo torna no competitivo en los mercados nacionales e internacionales especializados.

Basados en las cercanías de las personas dedicadas al comercio de esta hortaliza, el estado debe capacitar a este gremio en políticas de asociatividad en aras de incrementar los rendimientos por unidad de superficie y reducir las pérdidas por mala calidad y presentación de un producto altamente perecedero. 
El grupo IV es el de mejores prospectivas para incursionar en el mercado nacional e internacional, dado su grado de escolaridad, uso de la refrigeración y experiencia en el comercio con almacenes de cadena, por presentar mejor calidad de fruto.

\section{REFERENCIAS}

Agronet. 2009. Análisis - Estadísticas. Área Cosechada, Producción y Rendimiento de Berenjena, 1997-2009. Ministerio de Agricultura y Desarrollo Rural. http:// www.agronet.gov.co/www/htm 3 b/ ReportesAjax/VerReporte.aspx Febrero 2011]

Almansa, J. 2011. La singularidad y el empleo que genera la berenjena de Almagro son motivos suficientes para promocionar un alimento muy nuestro. http://www.lacerca.com/noticias/ castilla_la_mancha/berenjena_almagro_ promocionar_alimento-75983-1.html [7 Febrero 2011]

Bustamante, J. y Gálvez, J. 2010. Tipificación de los sistemas de producción de productores de leña certificada, de las comunas de Mariquina, Corral y Valdivia, en la Región de los Ríos. Bosque Nativo, 46:3-11.

Coca, A. y Solís, J. 2008. Posicionamiento de productos: Caso: vinos de altura en Bolivia. Compendium, 21: 1-20.

\section{Cordero-Salas, C., Chavarría, H., Echeverri, R.} y Sepúlveda, S. 2003. Territorios rurales, competitividad y desarrollo. Serie Cuadernos Técnicos, No 23. IICA.18p.
Cuadras, C. 1981. Análisis de coordenadas principales. En: Cuadras, C. (Ed), Métodos de análisis multivariante, Editorial EUNIBAR, Barcelona, p295344.

Cruz, C. 2004. Programa Genes Versao Windows: Aplicativo Computacional em Genética e Estatística. Viçosa: Univ. Fed. Viçosa. 648 p.

Cruz, C. y Carneiro, P. 2003. Modelos biométricos aplicados ao melhoramento genético. Viçosa: UFV, 585p.

Dos Santos, R. y Henz, G. 1993. Conservação pós- colheita da berinjela "Ciça"em função do horario da colheita. Pesq. agropec. bras. 28(5):563-565.

Espinosa, P., Játiva, P. y Suarez, G. 1990. Caracterización de los sistemas de producción agrícola de productores de maíz de la provincia de Bolívar en Ecuador. En: Escobar, G. y Berdegué, J. (Ed). Tipología de sistemas de producción agrícola. Red Internacional de Metodología de Investigación de Sistemas de Producción (RIMISP), Santiago de Chile, p157-166.

Escobar, G. y Berdegué, J. 1990. Tipificación de sistemas de producción agrícola. Red Internacional de Metodología de Investigación de Sistemas de Producción (RIMISP), Santiago de Chile. 282p.

FAO, 2010. Principales países exportadores de berenjena. http://faostat.fao.org. [6 Febrero 2011]. 
Ferrato, J. y Mondino, M. 2008. Producción, consumo y comercialización de hortalizas el mundo. Revista Agromensajes. Facultad de Ciencias Agrarias UNR. http://www.fcagr.unr.edu. ar [8 Febrero 2011].

Gower, J. 1966. Some distance properties of latent root and vector methods used in multivariate analysis. Biometrika 53(3):325-338.

Guajardo, R. y Ríos, M. 2009. Implicaciones de NAFTA, TLCUEM y de la integración de China a la OMC en el mercado de la berenjena: Una perspectiva de equilibrio especial. Economía Agraria y Recursos Naturales 9(1):91-109.

Hang, G., Kebat, C., Bravo, M., Larrañaga, G., Seibane, C., Ferraris, G., Otaño, M. y Blanco, V. 2010. Identificación de sistemas de producción hortícola en el Partido De La Plata, provincia de Buenos Aires, Argentina. Bioagro 22(1):81-86.

\section{IICA (Instituto Interamericano de Cooperación} para la Agricultura). 2007. Guía práctica para la exportación a Estados Unidos de berenjena. IICA, Managua-Nicaragua, 11p.

IWS, 2011. Área, producción y rendimiento de hortalizas en Colombia. http://www. prochile.cl. [8 Febrero 2011].

Jara, E., Modrego, F., Bedergue, J. y Celis, X. 2009. Empresas agrícolas en Chile: Caracterización e implicancias para las políticas de innovación y competitividad en el sector agroalimentario. Fundación para la Innovación Agraria - Centro Latinoamericano para el Desarrollo Rural. 26p.

Mendoza, G. 2002. Diagnóstico del mercadeo agrícola y agroindustrial en Colombia: una estrategia para la reactivación de la agricultura. $2^{\circ}$ Edición Fundación Universidad de Bogotá Jorge Tadeo Lozano. Santa Fe de Bogotá. Colombia, 208p.

MINAGRI (Ministerio de Agricultura de Chile). 2000. Una política de Estado para la Agricultura Chilena Período 2000-2010, Gobierno de Chile. http://www.lib. utexas.edu/benson [8 Febrero 2011].

Mineco - Unión Europea, 2010. Dinámica comercial de la berenjena. http://www. export.com.gt. [8 Febrero 2010].

Miranda, D. y Carranza, C. 2010. Caracterización de sistemas productivos de pasifloráceas n Colombia. Memorias I Congreso Latinoamericano de Passiflora. Corporación Centro de Investigación para la Gestión Tecnológica de Passiflora del Departamento del Huila y Asohofrucol. Neiva, noviembre de 2010, p27-59.

Miranda, E. 1990. Tipificación de los pequeños agricultores: Ejemplo de la metodología aplicada a los productores de frijol de Itarare, S. P., Brasil. En: Escobar, G. y Berdegué, J. (Ed). Tipología de sistemas de producción agrícola. Red Internacional de Metodología de Investigación de Sistemas de Producción (RIMISP), Santiago de Chile, p119-140. 
Mohammandi, S. y Prasanna, B. 2003. Analysis of Genetic Diversity in Crop Plants-Salient Statistical Tools and Considerations. Crop Sci. 43(4):12351248.

Poole, N. y Gomis, F. 2000. Análisis de las características productivas y de comercialización de los citricultores valencianos y su relación. Estudios Agrosociales y Pesqueros, 189:157-178.

Rao, C. 1952. Advances statistical methods in biometric research. New York: Jhon Willey and Sons.330p.

Ríos, G., Forero, C., Zuluaga, L., Echeverri, D., Botera, M., Franco, G., Álvarez, R. y García, J. 2007. Zonificación y caracterización de sistemas de producción de naranja común (Citrus sinensis Osbek) que utilizan criterios de BPA, en el departamento de Cundinamarca. http://200.75.42.3/ sitioweb/Archivos/oferta/Art.Cient._1_. pdfFINAL.pdf [24-Febrero 2011].
Rohlf, F. 1992. NTSYS-pc (Numerical Taxonomy and Multivariate Analysis System). Version 1.70. Exeter, Setauket, NY.

Salinas, Y. y Raigosa, B. 2005. Análisis de la situación de colombiana como productor de hortalizas en el mercado internacional. Agronomía, 13(2):7-22.

SIPSA. 2008. Costos asociados a la comercialización de berenjena. Sistema de Información de Precios del Sector Agropecuario-SIPSA, Bogotá, 4p.

Vicente, J. 2011. Análisis de coordenadas principales. http://biplot.usal.es/ DOCTORADO/3CICLO/BIENIO-04-06/ ACP/COORPRIN(apuntes).pdf $[6$ Febrero 2011]. 